\title{
The extent of retraction clefts correlates with lymphatic vessel density and VEGF-C expression and predicts nodal metastasis and poor prognosis in early-stage breast carcinoma
}

\author{
Geza Acs ${ }^{1,2,3,4}$, Gyorgy Paragh ${ }^{3}, Z_{\text {Zsuzsa Rakosy }}^{3}$, Christine Laronga ${ }^{2}$ and Paul J Zhang ${ }^{5}$ \\ ${ }^{1}$ Department of Anatomic Pathology, Moffitt Cancer Center, Tampa, FL, USA; ${ }^{2}$ Comprehensive Breast \\ Program, Department of Women's Oncology, Moffitt Cancer Center, Tampa, FL, USA; ${ }^{3}$ Experimental \\ Therapeutics Program, Moffitt Cancer Center, Tampa, FL, USA; ${ }^{4}$ Women's Pathology Consultants, Ruffolo \\ Hooper \& Associates, MD, PA, Tampa, FL, USA and ${ }^{5}$ Department of Pathology and Laboratory Medicine, \\ University of Pennsylvania School of Medicine, Philadelphia, PA, USA
}

\begin{abstract}
Although the earliest feature of disseminated disease in breast cancer is regional lymph node involvement, little is known about the mechanisms whereby cancer cells interact with lymphatic endothelial cells and enter the lymphatic system. We have previously reported that the extensive presence of retraction clefts in breast carcinomas highly significantly correlates with lymphatic tumor spread and predicts poor outcome, suggesting that retraction clefts are not just fixation artifacts, but real potential spaces that are exaggerated by tissue processing and may reflect an early stage of lymphatic invasion. In this study, we examined the correlation between the extent of retraction clefts and lymphangiogenesis, as assessed by lymphatic vessel density and vascular endothelial growth factor-C (VEGF-C) expression in a series of 256 early-stage breast carcinomas. The presence and extent of retraction clefts around tumor cell nests was determined by review of all hematoxylinand eosin-stained tumor sections. Lymphatic vessels were detected by podoplanin immunohistochemistry and lymphatic vessel density was measured using the hot-spot method. The expression of VEGF-C in the tumor cells was determined by immunohistochemistry and analyzed semiquantitatively on a four-tiered scale. High levels of retraction clefts, peritumor lymphatic vessel density and VEGF-C expression at the invasive edge in breast carcinomas significantly correlated with tumor size, histological grade, lymphatic invasion and nodal metastasis. Breast carcinomas showing extensive retraction clefts ( $>20 \%$ of tumor volume) were found to have significantly higher lymphatic vessel density and VEGF-C expression levels compared to tumors without this feature. High retraction clefts, peritumor lymphatic vessel density and VEGF-C expression predicted poor outcome in breast carcinomas. Our results support the hypothesis that retraction clefts are real potential spaces that may represent 'pre-lymphatic spaces' facilitating initial lymphatic invasion and that growth factors secreted by the tumor cells may stimulate tumor-associated lymphangiogenesis by promoting the endothelialization of these 'pre-lymphatic channels'.

Modern Pathology (2012) 25, 163-177; doi:10.1038/modpathol.2011.138; published online 21 October 2011
\end{abstract}

Keywords: breast carcinoma; lymphatic vessel density; podoplanin; retraction cleft; VEGF-C

Correspondence: Dr G Acs, MD, PhD, Department of Anatomic Pathology, Moffitt Cancer Center, 12902 Magnolia Drive, Tampa, FL 33612, USA.

E-mail: geza.acs@moffitt.org

Received 5 April 2011; revised 1 July 2011; accepted 4 July 2011; published online 21 October 2011
In most solid tumors, including breast cancer, the spread of cancer cells via the lymphatics to regional lymph nodes is an important early event in metastatic disease. ${ }^{1}$ Nodal metastasis is an important factor in the staging of breast cancers, an important prognostic indicator for outcome, and forms the basis of breast cancer treatment by surgery, adjuvant 
chemotherapy and radiation. ${ }^{1}$ Despite the major role for the lymphatics in the spread of cancers, little is known about the mechanisms whereby tumor cells enter the lymphatic system $^{2,3}$ and whether nodal metastasis is dependent on tumor-induced lymphangiogenesis or invasion of pre-existing lymphatic vessels. ${ }^{2}$

In recent years, several markers specific for lymphatic endothelium, including vascular endothelial growth factor receptor-3 (VEGFR-3), the transmembrane proteins LYVE-1 and podoplanin, and the transcription factor Prox-1, have been used to evaluate intratumor lymphatic vessels in solid tumors, including breast cancer. ${ }^{4-6}$ Intratumor lymphatic endothelial cells were shown to be capable of proliferation in some types of cancer, suggesting de novo lymphangiogenesis. ${ }^{4,7}$ These studies also showed a correlation between lymphatic vessel density and nodal metastasis in some tumors, ${ }^{8}$ but not in others. ${ }^{7}$ Nevertheless, the existence of lymphangiogenesis in breast carcinoma is one of the most controversial areas in the breast cancer literature. The significance of the presence and density of intra- and peritumor lymphatics for the survival of patients with breast cancer is currently unknown.

Research during recent years has also provided a better understanding of the molecular mechanisms underlying the development and maintenance of lymphatic vessels, and their role in various pathological conditions. ${ }^{1,9}$ Two lymphangiogenic growth factors named vascular endothelial growth factor-C (VEGF-C) and VEGF-D that signal through VEGFR-3 have been discovered. ${ }^{9}$ In experimental tumors, VEGF-C and VEGF-D expression has been shown to induce lymphangiogenesis and correlate with lymphatic invasion and lymph node metastasis. ${ }^{10-12}$ Other studies have also shown that overexpression of VEGF-C in breast cancer cells increases intra- and peritumor lymphangiogenesis and induces metastatic spread. ${ }^{11,13}$ VEGF-C expression in human breast cancer was also shown to be strongly associated with lymph node metastasis and unfavorable prognosis. ${ }^{14,15}$

We have recently reported that retraction clefts around tumor cell nests are commonly seen in invasive ductal (no special type) carcinomas of the breast. ${ }^{16,17}$ We have shown that the extent of retraction clefts correlates significantly with tumor size and histological grade, and shows a strong association with lymphatic invasion and nodal metastases. We have proposed that retraction clefts seen around tumor cell nests and glands in invasive ductal carcinomas are likely a morphological reflection of altered tumor-stromal interactions, which may contribute to tumor progression and lymphatic spread observed in these patients.

In this study, we examined whether the presence and extent of retraction clefts in invasive ductal carcinomas correlate with lymphangiogenesis as determined by VEGF-C expression and lymphatic vessel density, and lymphatic tumor spread in a large series of human breast carcinomas.

\section{Materials and methods}

\section{Clinical Samples and Clinical Data}

In all, 256 cases of previously untreated stage pT1 and pT2 primary unilateral invasive breast carcinomas were selected from the Surgical Pathology files of the University of Pennsylvania Medical Center. Patients were diagnosed between 1 January 1987 and 31 December 1996. All hematoxylin and eosin (H\&E)-stained slides were reviewed and the diagnoses were confirmed, including histological type and grade, based on the established criteria. ${ }^{18}$ The percentage of invasive carcinoma showing retraction clefts was estimated independently by two pathologists (GA and PJZ) based on all available H\&Estained tumor sections. Tumor cells, glands and nests surrounded by a clear space without an endothelial lining, which separated the tumor cells from the adjacent stroma, were considered as retraction clefts (Figure 1a). ${ }^{16,17}$ Tumors were evaluated to determine the presence or absence of lymphatic invasion based on all available tumor sections. The presence of lymphatic invasion and/or the lack of a lymphatic endothelial lining associated with retracted stroma was confirmed in each case by highlighting the lymphatic vessels using immunohistochemical stains for podoplanin (Figures 1b-f). ${ }^{19}$ Based on the results of prior studies, ${ }^{16,17}$ cases having retraction clefts involving at least $20 \%$ of the tumors were considered to show 'extensive retraction clefts'; this cutoff value also corresponded to the mean value for all tumors.

The clinicopathological features of the tumors are summarized in Table 1. Surgical treatment consisted of partial mastectomy/lumpectomy in 252 patients, whereas four patients underwent mastectomy. Axillary sentinel lymph node biopsy and/or complete axillary lymph node dissection was performed in all cases; the median number of lymph nodes examined per case was 15 (range 1-45). Nodal metastasis was present in $57(23 \%)$ patients; the median number of positive lymph nodes was 1 (range 1-15). Information regarding estrogen (ER) and progesterone (PR) receptor status was retrieved from the pathology reports. All patients received postoperative adjuvant radiation therapy. In total, $95(37 \%)$ patients received adjuvant chemotherapy and 128 (50\%) patients received hormone therapy in the form of Tamoxifen. Follow-up of patients was performed on the basis of information reported in the clinical histories. We considered as uncensored only records of patients who died of disease; we considered as censored records of all patients who were alive at follow-up or patients who died of a cause not related to the disease. Study protocols were approved by the University of Pennsylvania Institutional Review Board. 


\section{Immunohistochemistry}

Immunohistochemical assays were performed on 5 - $\mu$ m-thick formalin-fixed paraffin-embedded sections. For podoplanin and VEGF-C immunostaining, ${ }^{19}$ slides were boiled in $1 \times$ EDTA (LabVision, Fremont, CA, USA) or $0.01 \mathrm{~mol} / \mathrm{l}$ sodium citrate buffer ( $\mathrm{pH}$ 6.0) for $20 \mathrm{~min}$, respectively. Slides were incubated with the D2-40 (mouse monoclonal, 1:25 dilution; Signet Laboratories, Dedham, MA, USA) and VEGF-C (rabbit polyclonal, 1:30 dilution; Zymed Laboratories, South San Francisco, CA, USA) antibodies for $1 \mathrm{~h}$ on room temperature. Immunohistochemical staining was performed on a DAKOCytomation Autostainer using the EnVision + HRP DAB system (DAKOCytomation, Carpinteria, CA, USA), according to the manufacturer's recommendations. A negative control was carried out in each case by omission of the primary antibody. For
D2-40 and VEGF-C, slides of normal human tonsil and colonic adenocarcinoma known to show strong VEGF-C immunoreactivity were used as positive controls, respectively.

Cytoplasmic VEGF-C immunoreactivity in tumor cells was evaluated semiquantitatively on a fourtiered scale. The percentage of weakly, moderately and strongly staining cells was determined, and a score was calculated as follows: Score (out of maximum of 300) $=$ sum of $1 \times$ percentage of weak, $2 \times$ percentage of moderate and $3 \times$ percentage of strong staining. ${ }^{19}$ As the invasive edge of tumors is the site where lymphatic invasion is likely to occur, it was previously suggested that VEGF-C expression in the marginal portion of tumors may be more important than in the central area. ${ }^{19,20}$ Thus, we evaluated VEGF-C immunoreactivity separately at the marginal portion (defined as tumor cells located within $2 \mathrm{~mm}$ of the external edge) and in the center
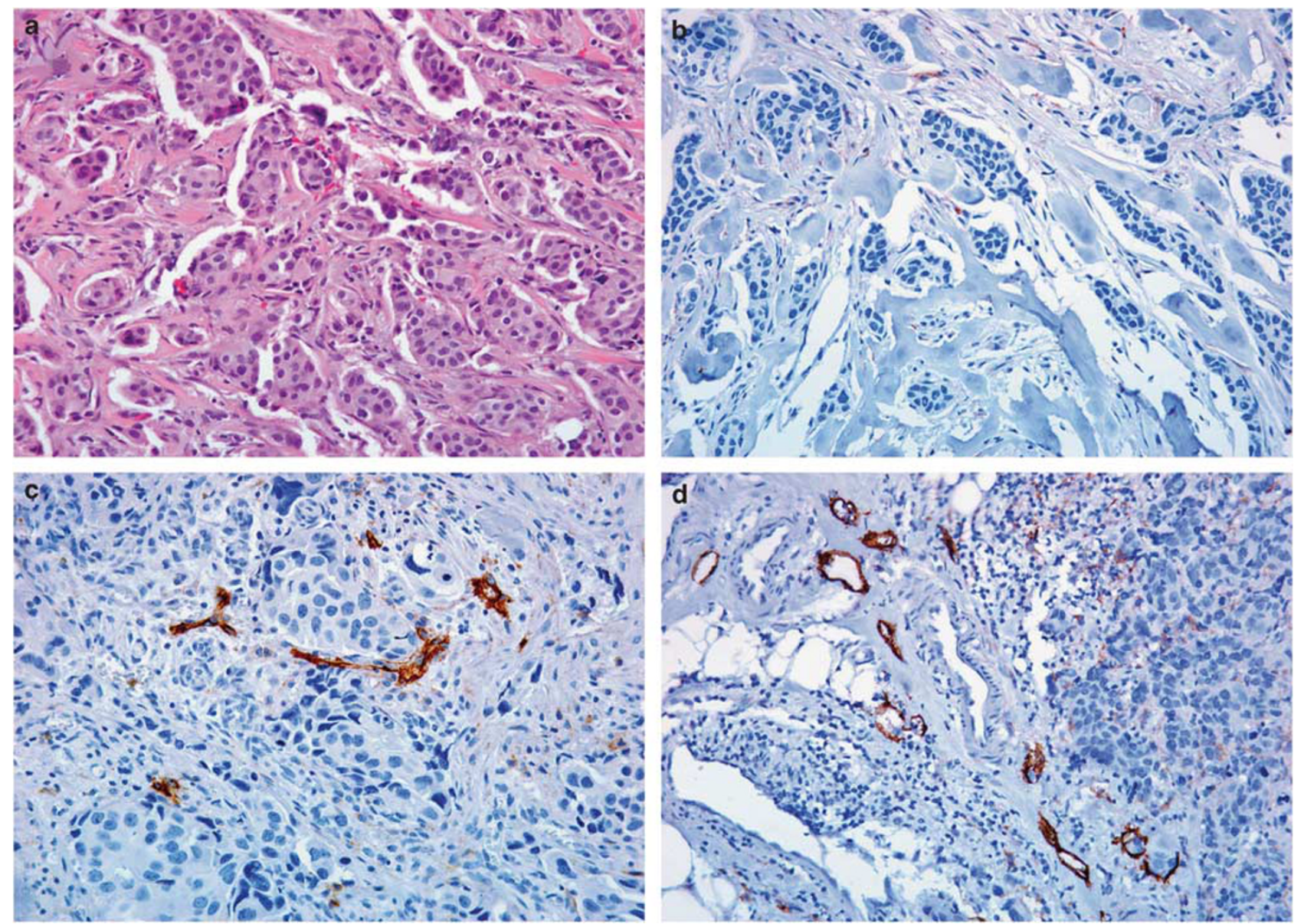

Figure 1 (a) Invasive ductal carcinoma showing prominent retraction clefts. The nests and cords of tumor cells are separated from the surrounding stroma by a clear space without an endothelial lining. (b) Immunohistochemical stain for podoplanin confirms the absence of an endothelial lining associated with the retraction clefts. (c, d) Intratumor lymphatic vessels (c) are characteristically small and flattened with a close lumen in contrast with the typically widely open, dilated lymphatics in peritumor regions (d) (immunohistochemical stains for podoplanin with hematoxylin counterstain). (e, f) Immunohistochemical stain for podoplanin highlights the presence of lymphatic invasion by tumor cells at the periphery of breast carcinomas (e) and occasionally within the tumor mass as well (f). (g) Immunohistochemical stain for podoplanin shows diffuse strong reactivity within the stroma of invasive breast carcinoma. (h) Invasive breast carcinoma showing strong vascular endothelial growth factor-C (VEGF-C) expression. Note the increased VEGF-C immunoreactivity in the tumor cells compared to the benign duct in the middle. 

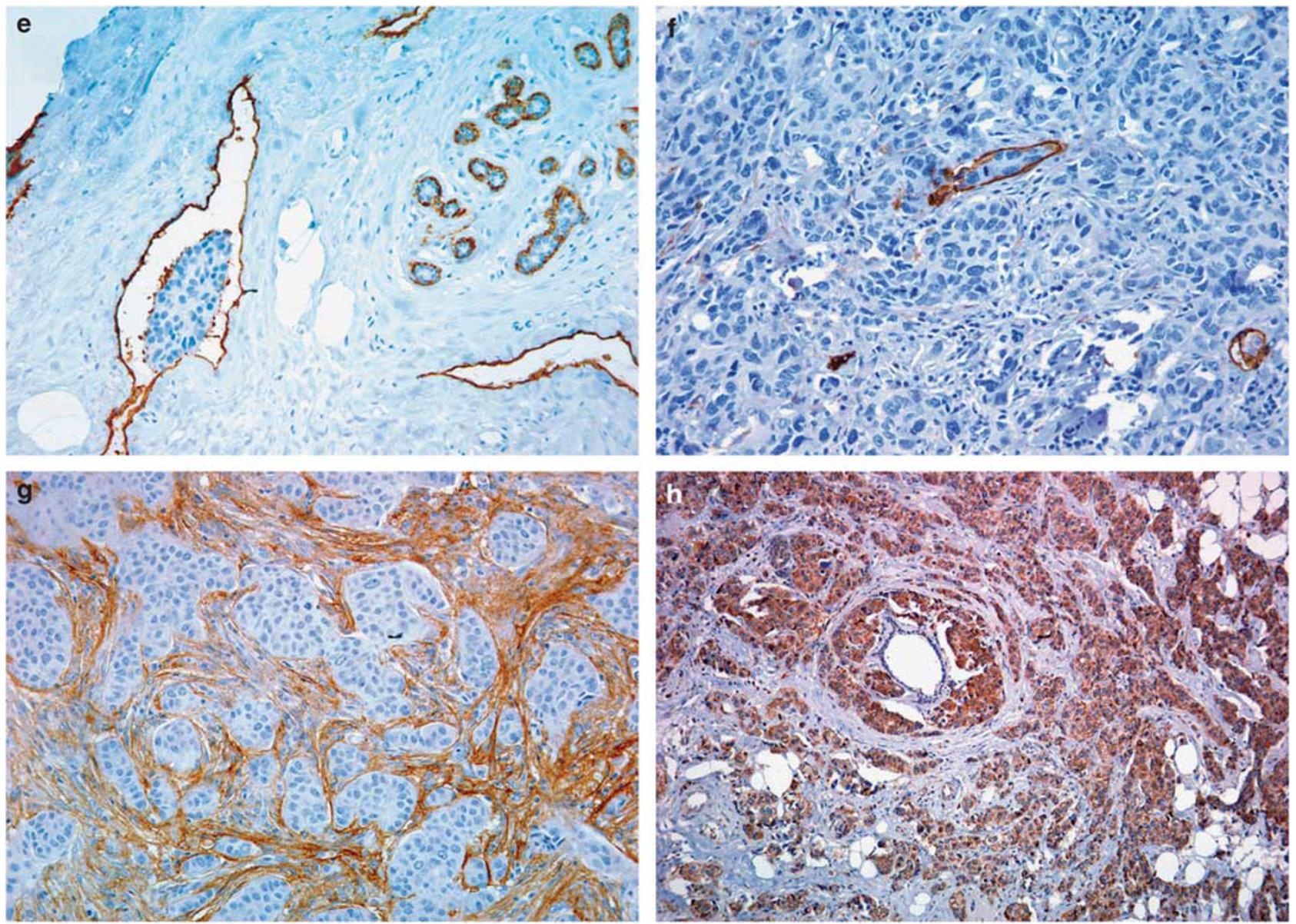

Figure 1 Continued.

(the rest of the tumor) of the tumors. High VEGF-C expression in the marginal and central portions of tumors was defined as scores higher than the respective median value for all tumors.

Intra- and peritumor lymphatic vessel densities were determined by the hot-spot method as described previously. ${ }^{19,21}$ Briefly, slides were scanned at low power, and intra- and peritumor areas with the highest density of podoplanin-positive vessels were identified. Intratumor lymphatic vessels were defined as those located within the tumor mass; most of such lymphatic vessels were present within the stroma in between tumor cell nests. Peritumor lymphatic vessels were those located outside of the tumor mass, but within $2 \mathrm{~mm}$ from the tumor edge. Lymphatic vessel density was determined by counting the number of podoplanin-positive vessels in five high-power $(\times 200)$ fields in the selected 'hotspot' areas, and the mean values of vessel counts were obtained. High intra- and peritumor lymphatic vessel density was defined as those higher than the respective mean value for all tumors.

Podoplanin reactivity in tumor cells and tumorassociated stroma was evaluated as present or absent; any level of staining was considered positive.

\section{Statistical Analysis}

Median lymphatic vessel density and VEGF-C immunostaining values were compared using the MannWhitney test or the Kruskal-Wallis one-way analysis of variance by ranks, followed by Dunn's multiple comparison test, when appropriate. Corresponding median intra- and peritumor lymphatic vessel density and VEGF-C expression values in the marginal and central portions of tumors were compared using the Wilcoxon signed-rank test. The correlation between percent retraction clefts, VEGF-C immunostaining and lymphatic vessel density values was estimated using the Spearman test. High vs low immunostaining and lymphatic vessel density values in carcinomas were compared using the unpaired $t$-test and the $\chi^{2}$ test, where appropriate. Survival curves were plotted using the method of Kaplan and Meier and compared using the log-rank test. A Cox proportional hazards model was used to assess the effect of tumor variables on survival. Statistical significance was determined if the two-sided $P$-value of a test was $<0.05$. Computations were performed using the GraphPad Prism (Version 5; GraphPad Software, San Diego, CA, USA) and SYSTAT (Version 10.2; SYSTAT Software, Richmond, CA, USA) softwares. 
Table 1 Summary of clinicopathological features $(n=256)$

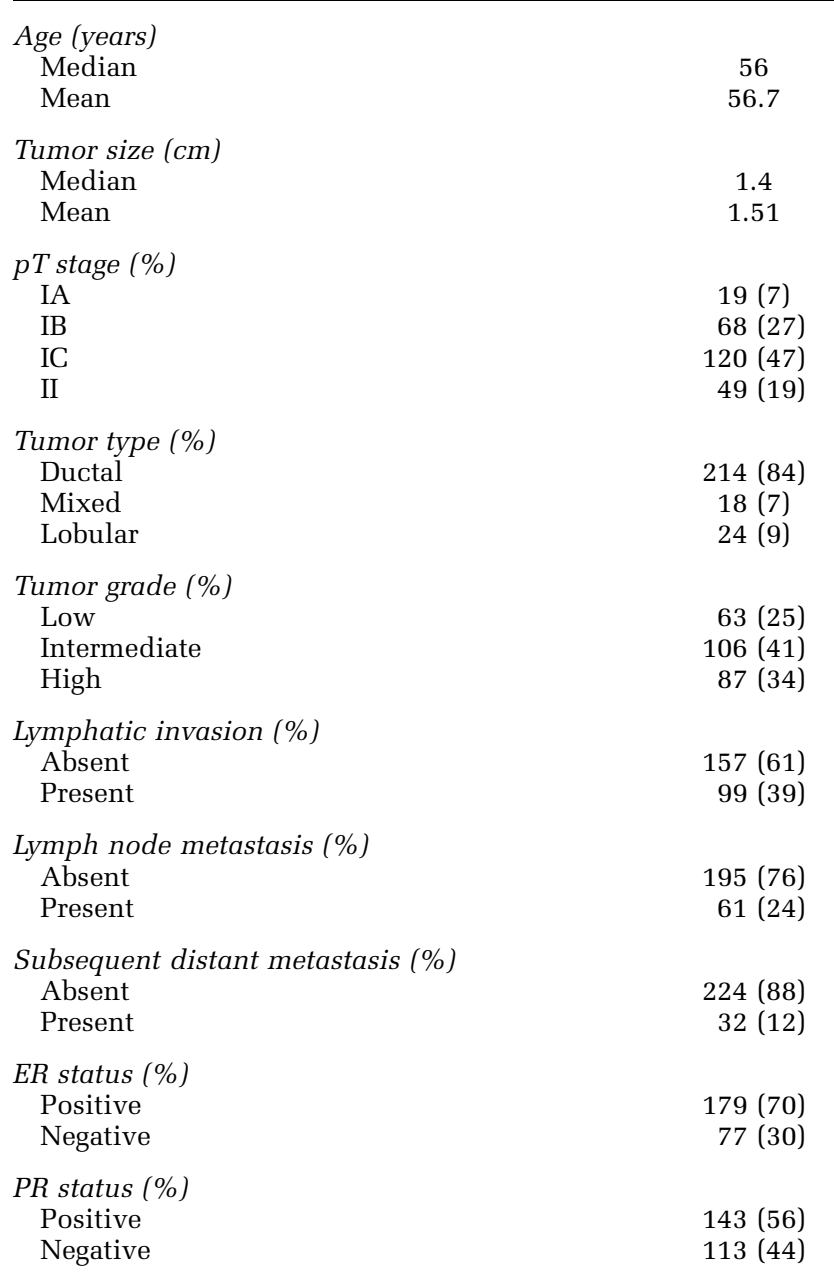

ER, estrogen receptor; PR, progesterone receptor.

\section{Results}

\section{Retraction Clefts}

Similar to prior results, ${ }^{16,17}$ variable degree of retraction clefts was present in 157 of 256 (61\%) invasive carcinomas (Figures 1a and b). The extent of retraction clefts in invasive tumors ranged from 0 to $90 \%$ with a median value of $10 \%(20.3 \pm 1.6$; mean \pm s.e.m.). As reported previously, ${ }^{16,17}$ the extent of retraction clefts showed a statistically highly significant correlation with tumor size, histological type and grade (Table 2). Similarly, tumors associated with lymphatic invasion and lymph node metastasis showed significantly higher percentage of retraction clefts compared to tumors without these features (Table 2).

\section{Lymphatic Vessel Density}

Intratumor lymphatic vessels were present in 116 of $256(45.1 \%)$ cases, whereas peritumor lymphatic
Table 2 Correlation of the extent of retraction clefts in invasive breast carcinomas with clinicopathological tumor features

\begin{tabular}{|c|c|c|c|c|}
\hline & \multirow[t]{2}{*}{$\mathrm{N}$} & \multicolumn{2}{|c|}{ Percent retraction clefts } & \multirow[t]{2}{*}{$\mathrm{P}$-value ${ }^{\mathrm{a}}$} \\
\hline & & Median & Mean \pm s.e.m. & \\
\hline \multicolumn{5}{|l|}{ Age (years) } \\
\hline$<50$ & 81 & 15 & $22.2 \pm 2.9$ & 0.1378 \\
\hline$\geq 50$ & 175 & 5 & $19.3 \pm 2.0$ & \\
\hline \multicolumn{5}{|l|}{ Туре } \\
\hline Ductal & 214 & 10 & $23.5 \pm 1.9$ & $<0.0001$ \\
\hline Mixed & 18 & 0 & $6.7 \pm 2.1$ & \\
\hline Lobular & 24 & 0 & $4.7 \pm 4.4$ & \\
\hline \multicolumn{5}{|l|}{ Grade } \\
\hline Low & 62 & 0 & $3.8 \pm 1.5$ & $<0.0001$ \\
\hline Intermediate & 107 & 10 & $20.4 \pm 2.4$ & \\
\hline High & 87 & 25 & $31.8 \pm 3.0$ & \\
\hline \multicolumn{5}{|l|}{ pT stage } \\
\hline IA & 19 & 0 & $8.2 \pm 4.7$ & $<0.0001$ \\
\hline IB & 68 & 0 & $11.3 \pm 2.4$ & \\
\hline IC & 120 & 17.5 & $23.8 \pm 2.4$ & \\
\hline II & 49 & 20 & $28.8 \pm 4.3$ & \\
\hline \multicolumn{5}{|c|}{ Lymphatic invasion } \\
\hline Absent & 157 & 0 & $8.1 \pm 1.3$ & $<0.0001$ \\
\hline Present & 99 & 30 & $39.5 \pm 2.6$ & \\
\hline \multicolumn{5}{|c|}{ Lymph node metastasis } \\
\hline Absent & 195 & 5 & $14.9 \pm 1.6$ & $<0.0001$ \\
\hline Present & 61 & 30 & $38.1 \pm 3.7$ & \\
\hline \multicolumn{5}{|l|}{ ER status } \\
\hline Positive & 179 & 5 & $20.1 \pm 2.0$ & 0.5816 \\
\hline Negative & 77 & 10 & $20.0 \pm 2.9$ & \\
\hline \multicolumn{5}{|l|}{ PR status } \\
\hline Positive & 143 & 5 & $19.9 \pm 2.2$ & 0.6949 \\
\hline Negative & 113 & 10 & $20.8 \pm 2.5$ & \\
\hline
\end{tabular}

ER, estrogen receptor; PR, progesterone receptor.

${ }^{\mathrm{a}}$ Kruskal-Wallis or Mann-Whitney test.

vessels were observed in 249 of 256 (97.3\%) cases. The podoplanin-positive lymph vessels were unevenly distributed throughout the tumors. The majority of intratumor lymph vessels were small and collapsed (Figure 1c). In contrast, lymphatic vessels located at the invasive edge of tumors were often enlarged and dilated (Figures $1 \mathrm{~d}$ and e). As expected, podoplanin immunostaining also highlighted the presence of lymphatic invasion, usually present at the periphery of tumors (Figure 1e). Less frequently, tumor cell emboli were also highlighted within intratumor lymphatic vessels as well (Figure 1f). Interestingly, in occasional cases, we identified partial podoplanin immunoreactivity associated with retraction clefts surrounding tumor cell nests, suggestive of partial endothelialization of the space surrounding the tumor cells (Figure 2). Although we found a significant positive correlation between the numbers of intra- and peritumor lymphatic vessels $(r=0.4808, P<0.0001$, Spearman test; Figure 3a), peritumor lymphatic vessel density $(5.64 \pm 0.28$, mean \pm s.e.m.) was significantly higher compared to 

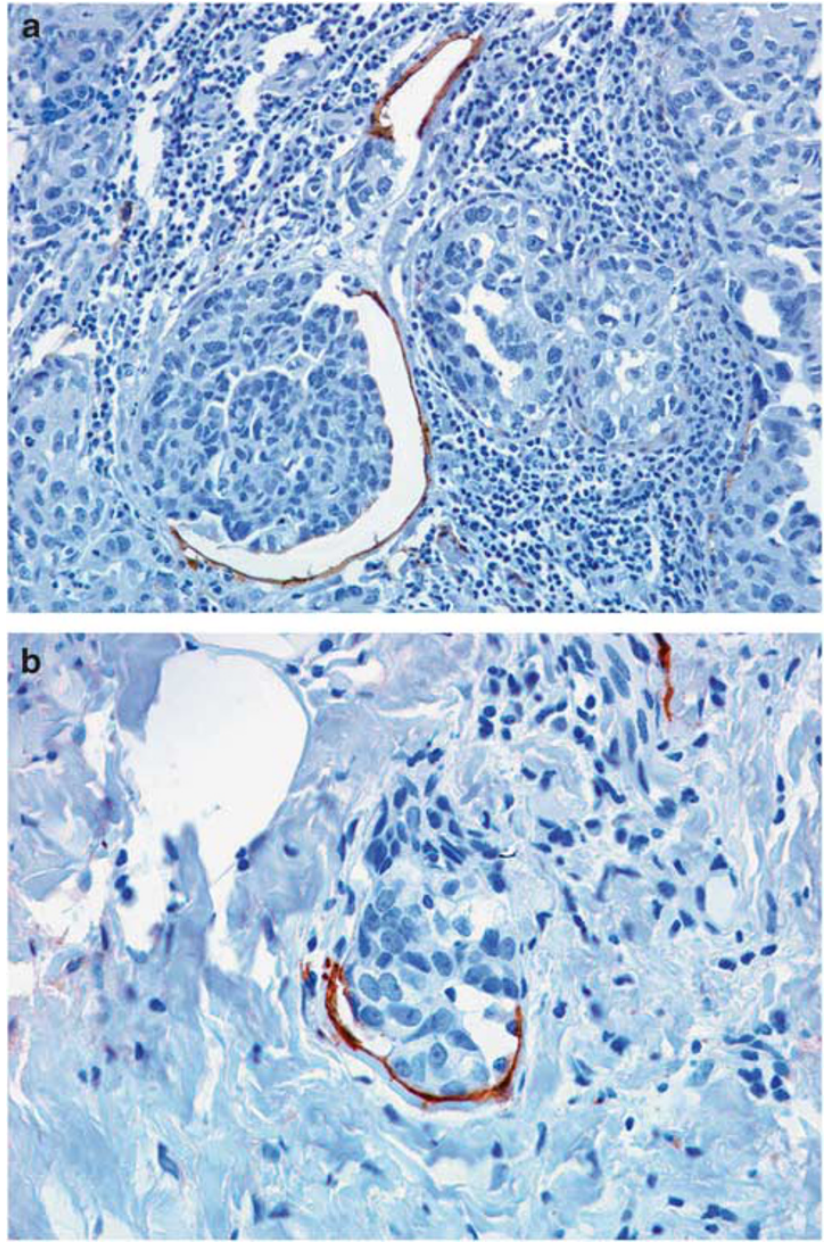

Figure 2 Partial immunostaining for podoplanin in association with retraction clefts surrounding clusters of tumor cells, suggestive of partial endothelialization of the 'pre-lymphatic spaces'.

intratumor lymphatic vessel density $(0.86 \pm 0.11$, mean \pm s.e.m.) in the corresponding tumors $(P<0.0001$, Wilcoxon signed-rank test; Figure $3 b)$.

The correlation of intra- and peritumor lymphatic vessel density with clinicopathological tumor features is summarized in Table 3 and Figures 3c-f. A significant positive association was seen between high intratumor lymphatic vessel density and histological grade, tumor size (pT stage), lymphatic invasion, nodal metastasis and ER status. Tumors of patients $<50$ years old showed significantly higher peritumor lymphatic vessel density compared with those of women 50 years of age or older. High peritumor lymphatic vessel density showed a statistically highly significant positive correlation with histological grade, tumor size, the presence of lymphatic invasion and nodal metastasis and lack of ER expression.

\section{VEGF-C Expression}

Immunohistochemical expression of VEGF-C was heterogeneous within the cancers; however, all but 19 tumors (93\%) showed at least focal weak VEGF-C immunoreactivity (Figure 1h). Although a positive correlation was seen between the levels of VEGF-C immunoreactivity in the tumor centers and peripheral areas $(r=0.9910, P<0.0001$, Spearman test; Figure 4a), the expression levels of VEGF-C were significantly higher at the infiltrating edge of cancers (median score of 100, mean \pm s.e.m.: $105.9 \pm 5.3$ ) compared to the central regions (median 30, mean \pm s.e.m.: $51.0 \pm 3.9, P<0.0001$, Wilcoxon signed-rank test; Figure 4b). Tumor cell groups within lymphatic spaces usually showed strong VEGF-C immunoreactivity (not shown).

The correlation of VEGF-C expression in the central area and infiltrating edge of breast carcinomas with clinicopathological tumor features is summarized in Table 4 and Figures 4c-f. A significant positive association was seen between high VEGF-C immunoreactivity in the central portion of tumors and histological grade and the presence of lymphatic invasion. Tumors of patients $<50$ years old showed significantly higher VEGF-C immunoreactivity at the infiltrating edge compared with those of women 50 years of age or older. High VEGF$\mathrm{C}$ expression at the infiltrating edge of breast carcinomas showed a statistically highly significant positive correlation with histological grade and the presence of lymphatic invasion, whereas the positive association with histological tumor type, tumor size (pT stage) and the presence of nodal metastasis was less strong.

\section{Podoplanin Expression in Tumor Cells and Tumor Stroma}

Podoplanin expression, ranging from focal weak to diffuse moderate cytoplasmic staining, was observed in the tumor cells in 5 of $256(2 \%)$ invasive breast carcinomas. All five tumors were ductal (no special type) carcinomas of intermediate or high histological grade. None of the five tumors was associated with lymphatic invasion or nodal metastasis. Podoplanin expression in tumor associated stroma was present in 136 (53\%) cases, ranging from focal weak to diffuse strong staining (Figure 1g). No immunoreactivity was observed in the stroma of benign breast tissue. The correlation between stromal podoplanin expression and clinicopathological tumor features in invasive breast carcinomas is summarized in Table 5. Stromal podoplanin expression showed a significant association with histological type, histological grade, tumor size (pT stage), presence of lymphatic invasion, ER status, extent of retraction clefts, intra- and peritumor lymphatic vessel density and VEGF-C expression both in the central portion and at the infiltrative edge of tumors.

\section{Correlation Among Retraction Clefts, Lymphatic Vessel Density and VEGF-C Expression}

We found a statistically significant positive correlation between the extent of retraction clefts in the 


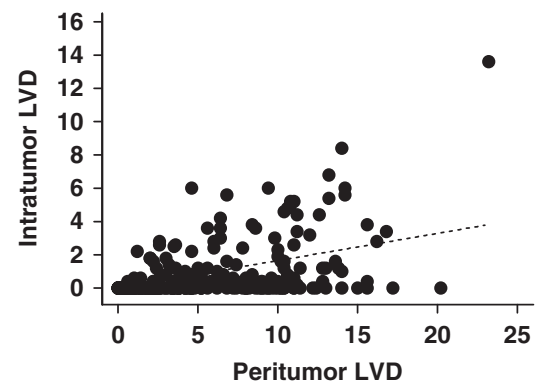

C

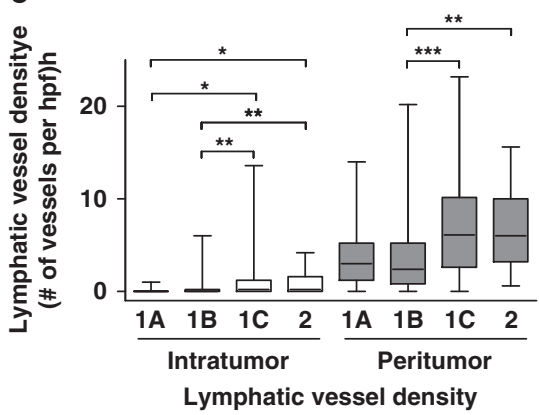

e
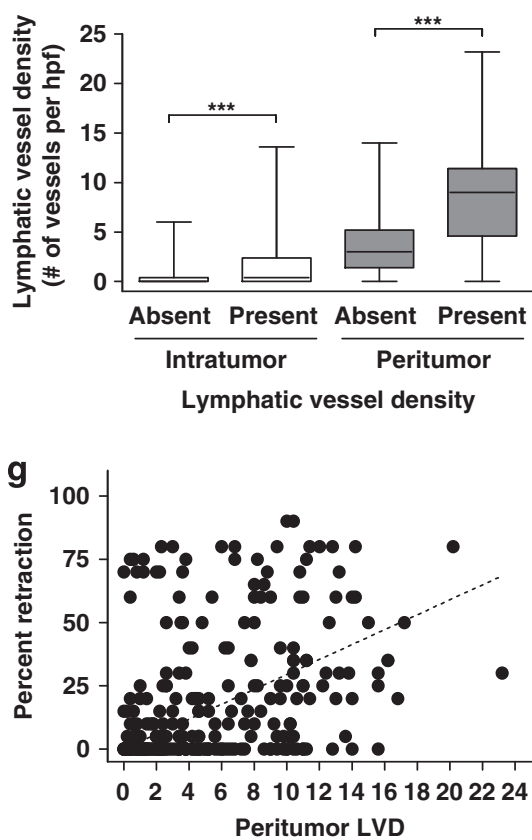

b

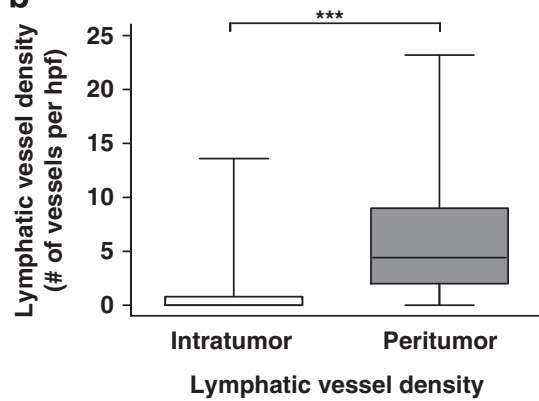

d
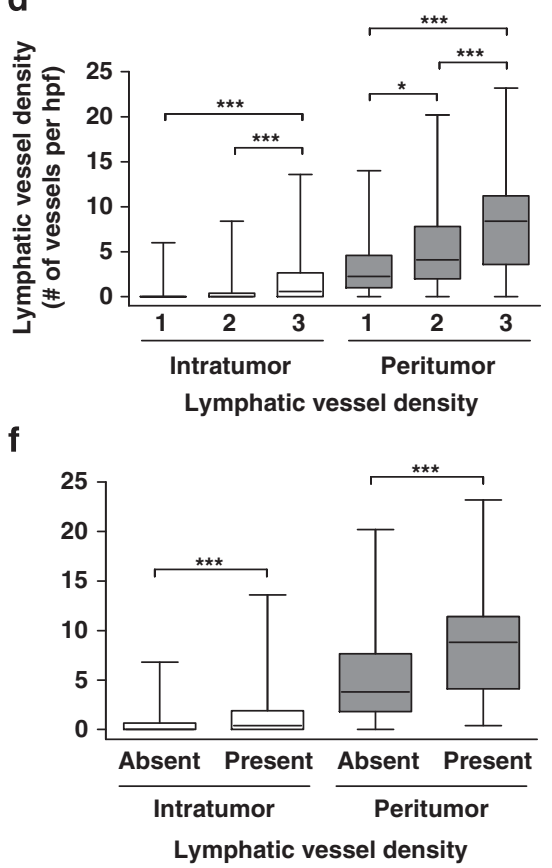

h

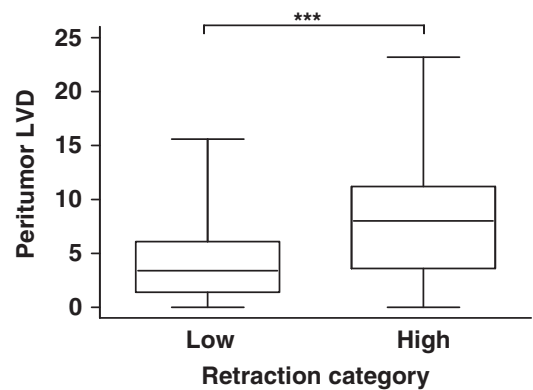

Figure 3 (a) There is a significant positive correlation between the numbers of intra- and peritumor lymphatic vessels in invasive breast carcinomas $(r=0.4808, P<0.0001$, Spearman test). The dotted line represents the calculated regression line. (b) Comparison of the numbers of intra- and peritumor lymphatic vessels in invasive breast carcinoma. (c-f) Comparison of the numbers of intra- and peritumor lymphatic vessels in invasive breast carcinomas according to tumor stage (c), histological grade (d), presence of lymphatic invasion (e) and lymph node metastasis (f). (g) There is a statistically significant positive correlation between the extent of retraction clefts and peritumor lymphatic vessel density in invasive breast carcinomas $(r=0.3545, P<0.0001$, Spearman test). The dotted line represents the calculated regression line. (h) Comparison of the number of peritumor lymphatic vessels in breast carcinomas showing low $(<20 \%) v s$ high (at least $20 \%$ ) levels of retraction clefts. $\left({ }^{*} P<0.05 ;{ }^{*} P<0.01\right.$; ${ }^{* *} P<0.0001$; Kruskal-Wallis or Mann-Whitney test).

tumors and intratumor $(r=0.1739, \quad P=0.0053$, Spearman test, not shown) and peritumor ( $r=0.3545, P<0.0001$, Spearman test; Figure 3g) lymphatic vessel density, and VEGF-C expression at the central portion $(r=0.4776, P<0.0001$, Spearman test, not shown) and invasive edge of carcinomas ( $r=0.5102, P<0.0001$, Spearman test; Figure 4g).
Invasive breast carcinomas showing extensive retraction clefts were associated with significantly higher intra- and peritumor lymphatic vessel density and showed significantly higher VEGF-C expression both at the central areas and at the infiltrating edge (Tables 3 and 4 and Figures $3 \mathrm{~h}$ and $4 \mathrm{~h})$. 
Table 3 Correlation of intra- and peritumoral lymphatic vessel density in invasive breast carcinomas with clinicopathological tumor features

\begin{tabular}{|c|c|c|c|c|c|c|c|}
\hline & \multirow[t]{2}{*}{$\mathrm{N}$} & \multicolumn{3}{|c|}{ Intratumoral lymphatic vessel density } & \multicolumn{3}{|c|}{ Peritumoral lymphatic vessel density } \\
\hline & & Median & Mean \pm s.e.m. & $\mathrm{P}$-value & Median & Mean \pm s.e.m. & $\mathrm{P}$-value ${ }^{\mathrm{a}}$ \\
\hline \multicolumn{8}{|l|}{ Age (years) } \\
\hline$<50$ & 81 & 0 & $0.95 \pm 0.18$ & 0.5915 & 6.2 & $6.85 \pm 0.54$ & 0.0061 \\
\hline$\geq 50$ & 175 & 0 & $0.82 \pm 0.13$ & & 3.8 & $5.08 \pm 0.32$ & \\
\hline \multicolumn{8}{|l|}{ Туре } \\
\hline Ductal & 214 & 0 & $0.95 \pm 0.12$ & 0.8727 & 4.6 & $5.90 \pm 0.32$ & 0.0984 \\
\hline Mixed & 18 & 0 & $0.38 \pm 0.16$ & & 2.4 & $3.26 \pm 0.56$ & \\
\hline Lobular & 24 & 0 & $0.66 \pm 0.22$ & & 3.7 & $4.95 \pm 0.79$ & \\
\hline \multicolumn{8}{|l|}{ Grade } \\
\hline Low & 63 & 0 & $0.35 \pm 0.14$ & $<0.0001$ & 2.25 & $3.30 \pm 0.41$ & $<0.0001$ \\
\hline Intermediate & 106 & 0 & $0.52 \pm 0.12$ & & 4.1 & $5.02 \pm 0.38$ & \\
\hline High & 87 & 0.6 & $1.66 \pm 0.24$ & & 8.4 & $8.08 \pm 0.53$ & \\
\hline \multicolumn{8}{|l|}{ pT stage } \\
\hline IA & 19 & 0 & $0.12 \pm 0.06$ & $<0.0001$ & 3.0 & $3.88 \pm 0.84$ & $<0.0001$ \\
\hline IB & 68 & 0 & $0.52 \pm 0.17$ & & 2.4 & $3.86 \pm 0.49$ & \\
\hline IC & 120 & 0.2 & $1.13 \pm 0.19$ & & 6.1 & $6.57 \pm 0.43$ & \\
\hline II & 49 & 0.2 & $0.91 \pm 0.17$ & & 6.0 & $6.60 \pm 0.63$ & \\
\hline \multicolumn{8}{|c|}{ Extent of retraction clefts } \\
\hline Low & 157 & 0 & $0.59 \pm 0.10$ & 0.0026 & 3.4 & $4.17 \pm 0.28$ & $<0.0001$ \\
\hline High & 99 & 0.2 & $1.30 \pm 0.22$ & & 8.0 & $7.96 \pm 0.50$ & \\
\hline \multicolumn{8}{|c|}{ Lymphatic invasion } \\
\hline Absent & 157 & 0 & $0.51 \pm 0.09$ & $<0.0001$ & 3.0 & $3.81 \pm 0.26$ & $<0.0001$ \\
\hline Present & 99 & 0.4 & $1.43 \pm 0.23$ & & 9.0 & $8.54 \pm 0.48$ & \\
\hline \multicolumn{8}{|c|}{ Lymph node metastasis } \\
\hline Absent & 195 & 0 & $0.73 \pm 0.10$ & 0.0158 & 3.8 & $4.94 \pm 0.30$ & $<0.0001$ \\
\hline Present & 61 & 0.4 & $1.40 \pm 0.32$ & & 8.8 & $8.33 \pm 0.65$ & \\
\hline \multicolumn{8}{|l|}{ ER status } \\
\hline Positive & 179 & 0 & $0.60 \pm 0.10$ & 0.0014 & 3.8 & $5.06 \pm 0.31$ & 0.0059 \\
\hline Negative & 77 & 0.2 & $1.46 \pm 0.26$ & & 5.6 & $6.93 \pm 0.57$ & \\
\hline \multicolumn{8}{|l|}{ PR status } \\
\hline Positive & 143 & 0 & $0.68 \pm 0.14$ & 0.1073 & 3.8 & $5.09 \pm 0.37$ & 0.0153 \\
\hline Negative & 113 & 0 & $1.10 \pm 0.16$ & & 5.0 & $6.33 \pm 0.43$ & \\
\hline
\end{tabular}

ER, estrogen receptor; PR, progesterone receptor.

${ }^{\mathrm{a}}$ Kruskal-Wallis or Mann-Whitney test.

We found no correlation between VEGF-C expression levels in the central regions and intratumor ( $r=0.2150, P=0.0604$, Spearman test, not shown) or peritumor $(r=0.1692, P=0.1413$, Spearman test, not shown) lymphatic vessel density. In contrast, a highly significant positive correlation was found between VEGF-C expression at the infiltrating edge of tumors and both intratumor $(r=0.3388$, $P<0.0001$, Spearman test) and peritumor $(r=0.6500, P<0.0001$, Spearman test) lymphatic vessel density.

\section{Survival Analysis}

During the follow-up interval, tumor recurrence was observed in $36(14 \%)$ cases, and $27(10.5 \%)$ patients died of disease. The median time to death for the uncensored subgroup was 68.3 (range 10.1-198.0) months, whereas the median follow-up of censored patients was 99.6 (range 3.9-210.4) months. The median time to tumor recurrence was 50.2 (range 4.8-155.4) months. In univariate analysis, histological grade $(P<0.0001)$, tumor size (pT stage, $P=0.010)$, presence of lymphatic invasion $(P<0.0001)$, lymph node metastasis $(P=0.005)$, extensive retraction clefts $(P<0.0001)$, intratumor lymphatic vessel density $(P=0.002)$, peritumor lymphatic vessel density $(P<0.0001)$, VEGF-C expression at the infiltrating edge $(P<0.0001)$ and $P R$ expression $(P=0.034)$ showed significant association with recurrence-free survival (Figures 5a, c and e). Histological grade $(P<0.0001)$, tumor size (pT stage, $P=0.002$ ), presence of lymphatic invasion 
a

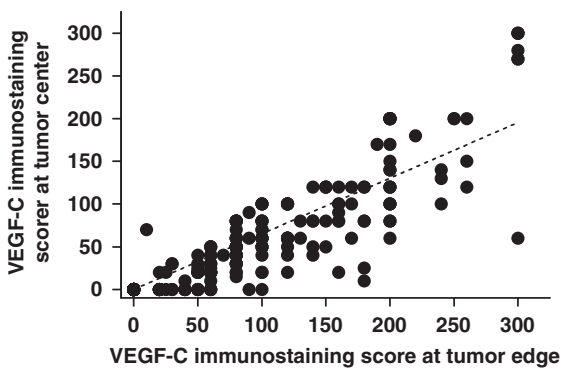

C

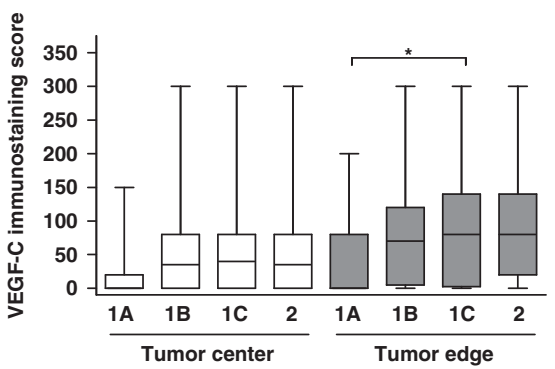

VEGF-C expression
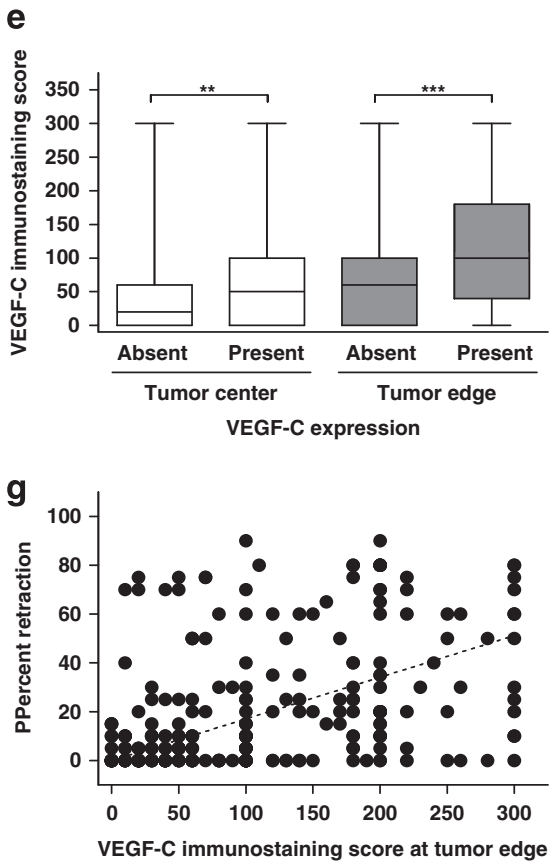

b
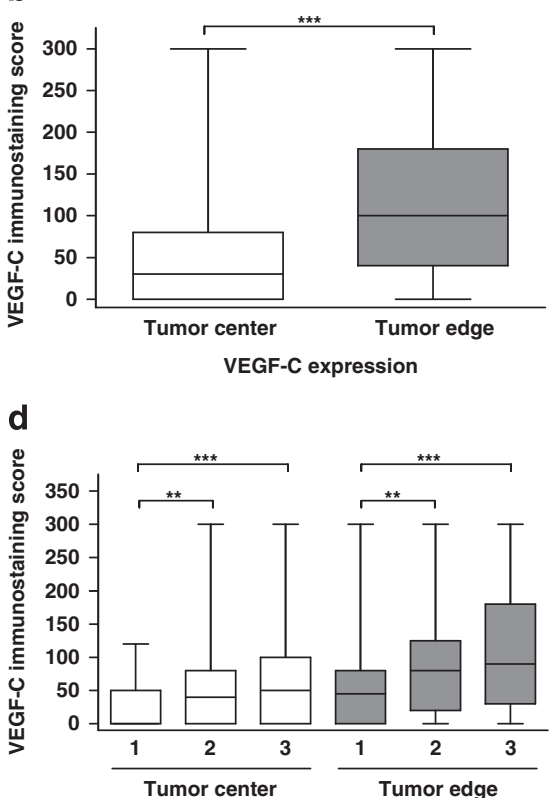

VEGF-C expression

f
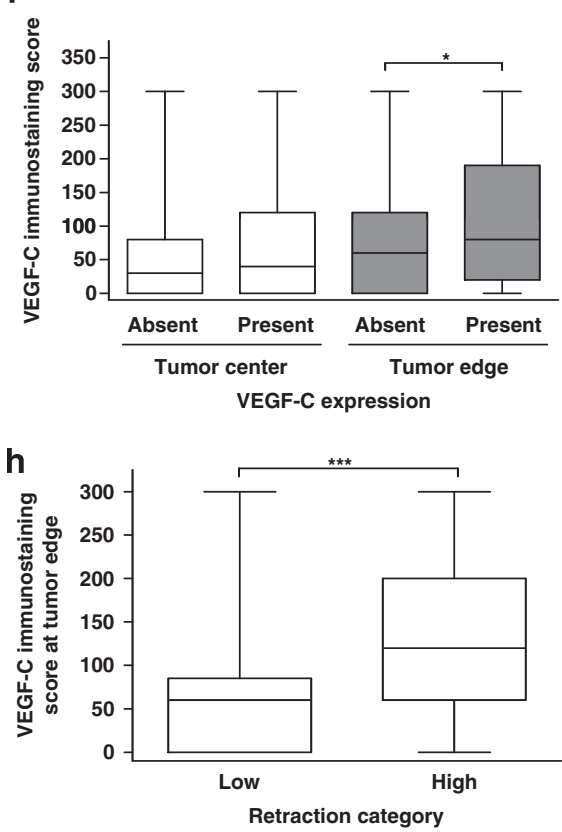

Figure 4 (a) There is a significant positive correlation between immunohistochemical expression of vascular endothelial growth factor-C (VEGF-C) in the center and peripheral portions of invasive breast carcinomas $(r=0.9910, P<0.0001$, Spearman test). The dotted line represents the calculated regression line. (b) Comparison of the levels of VEGF-C expression at the central and peripheral portions of invasive breast carcinomas as detected by immunohistochemistry. (c-f) Comparison of VEGF-C immunohistochemical expression levels in the central and peripheral portions of invasive breast carcinomas according to tumor stage (c), histological grade (d), presence of lymphatic invasion (e) and lymph node metastasis (f). (g) There is a statistically significant positive correlation between the extent of retraction clefts and the level of VEGF-C expression at the invasive edge of breast carcinomas $(r=0.5102, P<0.0001$, Spearman test). The dotted line represents the calculated regression line. (h) Comparison of the levels of immunohistochemical expression of VEGF-C at the invasive edge of breast carcinomas showing low $(<20 \%)$ vs high (at least $20 \%$ ) levels of retraction clefts. $\left({ }^{*} P<0.05\right.$; ${ }^{* *} P<0.01$; ${ }^{* * *} P<0.0001$; Kruskal-Wallis or Mann-Whitney test).

$(P<0.0001)$, lymph node metastasis $(P=0.031)$, extensive retraction clefts $(P<0.0001)$, PR expression $(P=0.005)$, peritumor lymphatic vessel density $(P=0.003)$, intratumor lymphatic vessel density $(P=0.02)$ and VEGF-C expression at the infiltrating edge of tumors $(P=0.001)$ were associated with poor overall survival (Figures $5 \mathrm{~b}, \mathrm{~d}$ and $\mathrm{f}$ ). In a subgroup analysis, interestingly, recurrence-free and overall survival of patients whose tumors were not associated with lymphatic invasion, but showed high 
Table 4 Correlation of VEGF-C expression in invasive breast carcinomas with clinicopathological tumor features

\begin{tabular}{|c|c|c|c|c|c|c|c|}
\hline & \multirow[t]{2}{*}{$\mathrm{N}$} & \multicolumn{3}{|c|}{$V E G F-C$ expression in tumor center } & \multicolumn{3}{|c|}{ VEGF-C expression at infiltrating edge } \\
\hline & & Median & Mean \pm s.e.m. & $\mathrm{P}$-value ${ }^{\mathrm{a}}$ & Median & Mean \pm s.e.m. & P-value ${ }^{\mathrm{a}}$ \\
\hline \multicolumn{8}{|l|}{ Age (years) } \\
\hline$<50$ & 81 & 75 & $89.6 \pm 15.0$ & 1.000 & 100 & $125.3 \pm 9.9$ & 0.0185 \\
\hline$\geq 50$ & 175 & 80 & $93.9 \pm 12.2$ & & 80 & $96.8 \pm 6.2$ & \\
\hline \multicolumn{8}{|l|}{ Туре } \\
\hline Ductal & 214 & 80 & $91.8 \pm 9.7$ & 0.9583 & 100 & $111.3 \pm 5.8$ & 0.0150 \\
\hline Mixed & 18 & 80 & $73.6 \pm 8.7$ & & 40 & $51.8 \pm 9.6$ & \\
\hline Lobular & 24 & 80 & $94.6 \pm 22.4$ & & 50 & $96.3 \pm 19.1$ & \\
\hline \multicolumn{8}{|l|}{ Grade } \\
\hline Low & 63 & 0 & $25.1 \pm 4.5$ & 0.0003 & 45 & $48.7 \pm 7.3$ & $<0.0001$ \\
\hline Intermediate & 106 & 40 & $55.9 \pm 6.2$ & & 80 & $87.1 \pm 7.2$ & \\
\hline High & 87 & 50 & $64.9 \pm 7.9$ & & 90 & $109.2 \pm 9.6$ & \\
\hline \multicolumn{8}{|l|}{ pT stage } \\
\hline IA & 19 & 0 & $22.9 \pm 9.8$ & 0.1243 & 0 & $40.3 \pm 13.5$ & 0.0489 \\
\hline IB & 68 & 35 & $55.6 \pm 8.3$ & & 70 & $86.6 \pm 9.8$ & \\
\hline IC & 120 & 40 & $51.1 \pm 5.4$ & & 80 & $88.3 \pm 7.1$ & \\
\hline II & 49 & 35 & $55.4 \pm 10.3$ & & 80 & $89.9 \pm 12.2$ & \\
\hline \multicolumn{8}{|c|}{ Extent of retraction clefts } \\
\hline Low & 157 & 50 & $50.2 \pm 7.5$ & $<0.0001$ & 60 & $58.4 \pm 4.8$ & $<0.0001$ \\
\hline High & 99 & 130 & $140.3 \pm 14.8$ & & 120 & $127.1 \pm 8.8$ & \\
\hline \multicolumn{8}{|c|}{ Lymphatic invasion } \\
\hline Absent & 157 & 20 & $40.8 \pm 4.2$ & 0.0065 & 60 & $66.4 \pm 5.4$ & $<0.0001$ \\
\hline Present & 99 & 50 & $67.1 \pm 7.6$ & & 100 & $113.2 \pm 8.8$ & \\
\hline \multicolumn{8}{|c|}{ Lymph node metastasis } \\
\hline Absent & 195 & 30 & $46.6 \pm 4.0$ & 0.2821 & 60 & $76.6 \pm 5.2$ & 0.0211 \\
\hline Present & 61 & 40 & $67.6 \pm 11.1$ & & 80 & $112.7 \pm 12.8$ & \\
\hline \multicolumn{8}{|l|}{ ER status } \\
\hline Positive & 179 & 60 & $90.4 \pm 12.4$ & 0.5085 & 80 & $100.6 \pm 6.2$ & 0.1848 \\
\hline Negative & 77 & 80 & $95.7 \pm 14.6$ & & 100 & $117.8 \pm 10.2$ & \\
\hline \multicolumn{8}{|l|}{ PR status } \\
\hline Positive & 143 & 65 & $92.3 \pm 13.6$ & 0.7231 & 70 & $96.9 \pm 6.9$ & 0.0573 \\
\hline Negative & 113 & 80 & $92.4 \pm 13.2$ & & 100 & $117.3 \pm 8.1$ & \\
\hline
\end{tabular}

ER, estrogen receptor; PR, progesterone receptor; VEGF, vascular endothelial growth factor.

${ }^{\mathrm{a}}$ Kruskal-Wallis or Mann-Whitney test.

retraction clefts $(n=21)$, was significantly worse than those with no lymphatic invasion and low retraction clefts $(n=136, P=0.0065$ and 0.0230 , respectively), and similar to those whose tumors were associated with overt lymphatic invasion ( $n=99, P=0.3912$ and 0.5234 , respectively) (Figures $5 \mathrm{~g}$ and $\mathrm{h}$ ).

For stepwise logistic regression, we included in the models tumor size, histological grade, lymphatic invasion, nodal metastasis and ER and PR status. Retraction clefts, lymphatic vessel density and VEGF-C expression were included in the model as binominal (low vs high) variants. Backward elimination for Cox regression led to a model with four independent terms predictive of overall survival (lymph node metastasis, $P=0.006$; extensive retraction clefts, $P=0.008$; histological grade, $P=0.068$; and tumor size, $P=0.07$ ), and three independent terms predictive of recurrence-free survival (lymph node metastasis, $P=0.008$, extensive retraction clefts, $P=0.009$ and histological grade, $P=0.057$ ).

\section{Discussion}

The main findings of this study are that the extensive presence of retraction clefts in early-stage invasive ductal carcinomas of the breast highly significantly correlates with lymphangiogenesis (as measured by lymphatic vessel density and VEGF-C expression) and lymphatic tumor spread (lymphatic invasion and nodal metastasis) and predicts a poor outcome. Our results also suggest that the clinical and prognostic significance of extensive retraction clefts is likely similar to those of overt lymphatic invasion and that retraction clefts may in fact represent an early stage of lymphatic invasion. However, it is important to note that in our cohort, 
Table 5 Correlation of podoplanin immunoreactivity in tumor stroma of invasive breast carcinomas with clinicopathological tumor features

\begin{tabular}{|c|c|c|c|}
\hline & \multicolumn{2}{|c|}{$\begin{array}{l}\text { D2-40 immunostaining } \\
\text { in tumor stroma }\end{array}$} & \multirow[t]{2}{*}{ P-value } \\
\hline & Present & Absent & \\
\hline \multicolumn{4}{|l|}{ Age (years) } \\
\hline$<50$ & 49 & 32 & \multirow[t]{2}{*}{$0.1383^{\mathrm{a}}$} \\
\hline$\geq 50$ & 87 & 88 & \\
\hline \multicolumn{4}{|l|}{ Tуре } \\
\hline Ductal & 123 & 91 & \multirow[t]{3}{*}{$0.0066^{\mathrm{a}}$} \\
\hline Mixed & 5 & 13 & \\
\hline Lobular & 8 & 16 & \\
\hline \multicolumn{4}{|l|}{ Grade } \\
\hline Low & 23 & 39 & \multirow[t]{3}{*}{$<0.0001^{\mathrm{a}}$} \\
\hline Intermediate & 39 & 68 & \\
\hline High & 74 & 13 & \\
\hline \multicolumn{4}{|l|}{ pT stage } \\
\hline $1 \mathrm{~A}$ & 5 & 14 & \multirow[t]{4}{*}{$0.0072^{\mathrm{a}}$} \\
\hline 1B & 29 & 39 & \\
\hline $1 \mathrm{C}$ & 73 & 47 & \\
\hline 2 & 29 & 20 & \\
\hline \multicolumn{4}{|c|}{ Lymphatic invasion } \\
\hline Absent & 74 & 83 & \multirow[t]{2}{*}{$0.0204^{\mathrm{a}}$} \\
\hline Present & 62 & 37 & \\
\hline \multicolumn{4}{|c|}{ Lymph node metastasis } \\
\hline Absent & 101 & 96 & \multirow[t]{2}{*}{$0.3006^{\mathrm{a}}$} \\
\hline Present & 35 & 24 & \\
\hline \multicolumn{4}{|l|}{ ER status } \\
\hline Positive & 80 & 97 & \multirow[t]{2}{*}{$0.0001^{\mathrm{a}}$} \\
\hline Negative & 56 & 23 & \\
\hline \multicolumn{4}{|l|}{ PR status } \\
\hline Positive & 73 & 70 & \multirow[t]{2}{*}{$0.5284^{\mathrm{a}}$} \\
\hline Negative & 63 & 50 & \\
\hline \multicolumn{4}{|c|}{ Extent of retraction clefts (\%) } \\
\hline Median & 20 & 0 & \multirow[t]{2}{*}{$<0.0001^{\mathrm{b}}$} \\
\hline Mean \pm s.e.m. & $26.1 \pm 2.3$ & $14.2 \pm 2.2$ & \\
\hline \multicolumn{4}{|c|}{ Intratumor lymphatic vessel density } \\
\hline Median & 0.2 & 0 & \multirow[t]{2}{*}{$0.0002^{\mathrm{b}}$} \\
\hline Mean \pm s.e.m. & $1.24 \pm 0.18$ & $0.44 \pm 0.09$ & \\
\hline \multicolumn{4}{|c|}{ Peritumor lymphatic vessel density } \\
\hline Median & 6.4 & 3.4 & \multirow[t]{2}{*}{$<0.0001^{\mathrm{b}}$} \\
\hline Mean \pm s.e.m. & $6.8 \pm 0.4$ & $4.3 \pm 0.3$ & \\
\hline \multicolumn{4}{|c|}{ VEGF-C at tumor center } \\
\hline Median & 50 & 20 & $0.0015^{\mathrm{b}}$ \\
\hline Mean \pm s.e.m. & $61.5 \pm 5.8$ & $39.0 \pm 5.2$ & \\
\hline$V E G F-C$ at infilt & g edge & & \\
\hline Median & 85 & 50 & $<0.0001^{\mathrm{b}}$ \\
\hline Mean \pm s.e.m. & $103.2 \pm 7.1$ & $63.3 \pm 6.3$ & \\
\hline
\end{tabular}

ER, estrogen receptor; PR, progesterone receptor; VEGF, vascular endothelial growth factor.

${ }^{\mathrm{a}} \chi^{2}$ test.

${ }^{\mathrm{b}}$ Kruskal-Wallis or Mann-Whitney test. the patients were not treated uniformly with regard to chemo- and hormonal therapy and validation of our findings with regard to the outcome in a cohort of patients treated uniformly would be important.

Tumor-associated lymphatic vessels are considered as the main route for tumor cell spread to axillary lymph nodes ${ }^{22}$ and lymphatic invasion by tumor cells is thought to be a prerequisite for dissemination via the lymphatic system. However, there has been considerable debate in the literature as to whether tumors can induce lymphangiogenesis, whether lymphatic invasion requires newly formed lymphatic channels or tumor cells invade pre-existing lymphatic vessels and whether lymphatic invasion require active intravasation by tumor cells through the walls of lymphatic vessels. ${ }^{1,4,6,10,11,22-25}$ It has recently been shown that the growth of new lymphatic vessels is controlled by VEGF-C and VEGF-D and their receptor, VEGFR-3. ${ }^{1,9}$ In experimental breast cancer models, the overexpression of VEGF-C was shown to strongly promote the growth of tumor-associated lymphatic vessels and enhance the spread of breast cancer cells to regional lymph nodes, indicating that these vessels can indeed serve as a conduit for lymphatic dissemination. ${ }^{11-13}$ Although the significance of lymphatic vessel density is still unclear, it was proposed that tumor cells exposed to more lymphatic vessels are more likely to spread to lymph nodes and to distant sites. ${ }^{21}$

Similar to previous studies, ${ }^{6,26}$ we found that the presence of intratumor lymphatic vessels could be demonstrated in almost $50 \%$ of invasive breast carcinomas; however, the number of peritumor lymphatic vessels was significantly higher. As previously described in cervical and other cancers, ${ }^{5,19}$ intratumor lymphatic vessels were characteristically small and flattened with a close lumen in contrast with the typically widely open, dilated lymphatics in peritumor regions. Although lymphatic invasion by tumor cells was rarely seen within the tumor mass, it was usually much more prominent involving the dilated peritumor lymphatic vessels. Our results also suggest that high peritumor lymphatic vessel density shows a stronger association with aggressive tumor features compared to intratumor lymphatic vessel density, and it appears to be a highly significant factor predicting poor overall and recurrence-free survival in our cohort of patients. High peritumor lymphatic vessel density was also associated with the presence of nodal metastases and aggressive behavior in several, but not all, other studies as well. ${ }^{6,19,24,27,28}$

Similar to prior reports, ${ }^{15}$ we detected VEGF-C expression in $93 \%$ of invasive breast carcinomas in our current series. We found that VEGF-C immunoreactivity was increased at the periphery of breast cancers consistent with the upregulation of VEGF-C expression at the invasive edge of the tumors as previously reported in breast, cervix and pancreatic carcinomas. ${ }^{19,20,24}$ Despite the abundance of data 

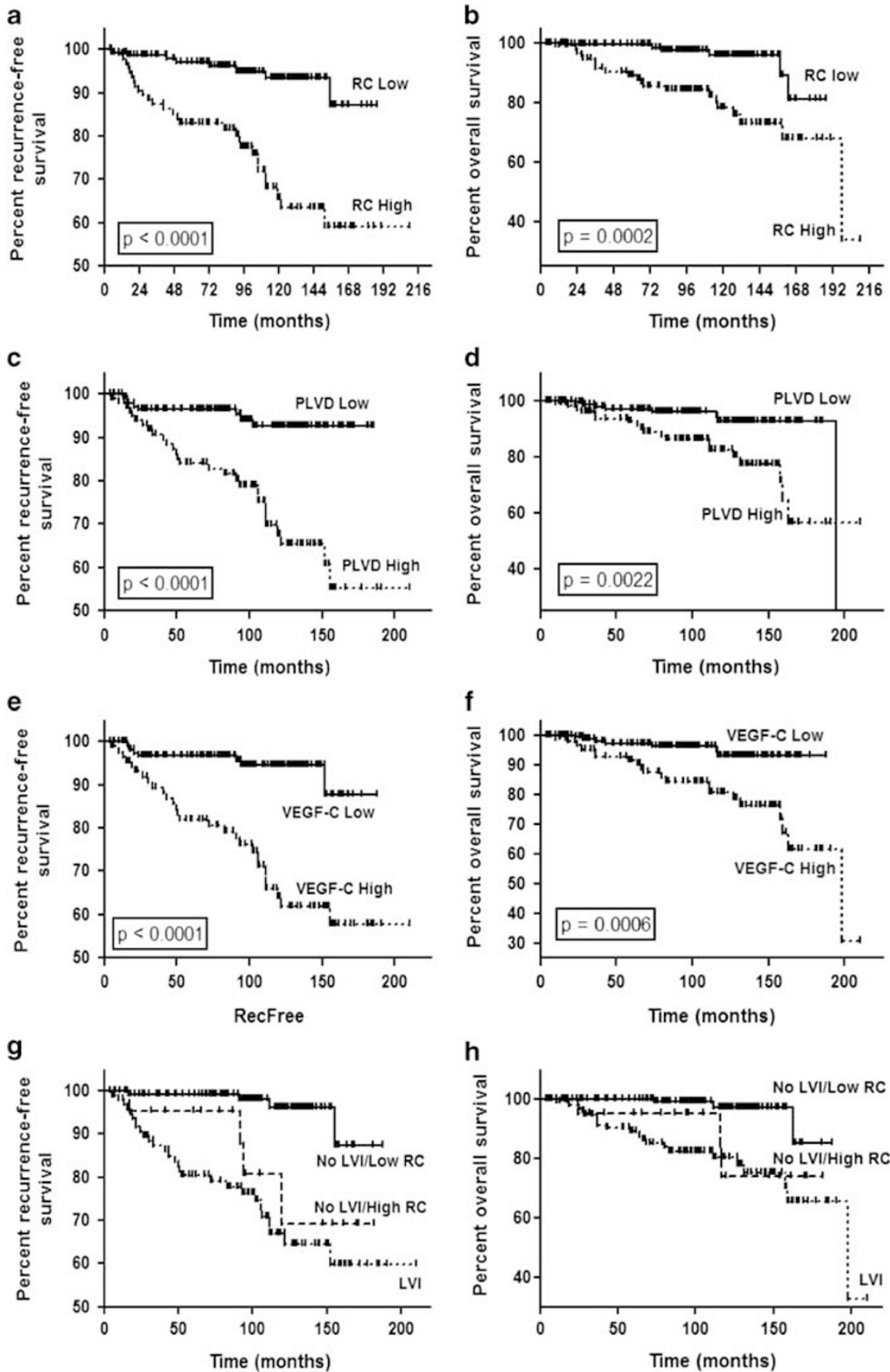

Figure 5 Kaplan-Meier recurrence-free $(\mathbf{a}, \mathbf{c}, \mathbf{e}, \mathbf{g})$ and disease-free $(\mathbf{b}, \mathbf{d}, \mathbf{f}, \mathbf{h})$ survival curves in invasive breast carcinomas stratified for low vs high levels of retraction clefts (a, b), low vs high peritumor lymphatic vessel density (c, d), low vs high vascular endothelial growth factor-C (VEGF-C) expression at the invasive edge of tumors (e, f) and low levels of retractions clefts and no lymphatic invasion vs high levels of retraction clefts and no lymphatic invasion vs the presence of lymphatic invasion (g, h) seen in the tumors. (g, h) Invasive breast carcinomas showing low levels of retraction clefts and no lymphatic invasion were associated with significantly better recurrencefree and overall survival compared to carcinomas showing high levels of retractions clefts and no lymphatic invasion $(P=0.0065$ and 0.023, respectively, Mantel-Cox log-rank test) and those showing overt lymphatic invasion $(P<0.0001$ and 0.0003 , respectively, MantelCox log-rank test). No difference in recurrence-free or overall survival was seen between invasive breast carcinomas showing high levels of retractions clefts and no lymphatic invasion vs those showing overt lymphatic invasion $(P=0.3912$ and 0.5234 , respectively, MantelCox log-rank test).

from animal tumor models showing that the VEGF-C/VEGF-D/VEGFR-3 axis promotes tumorassociated lymphangiogenesis and metastatic spread, ${ }^{10,11}$ this relationship is less conspicuous in human tumors where there is as yet little evidence for direct lymphangiogenesis. In recent years, a number of clinical studies have reported VEGF-C expression in human tumors and illustrated a 
significant association between VEGF-C levels of primary tumors and lymph node metastasis. ${ }^{11,29}$ Our results suggest that there is a strong correlation between VEGF-C expression at the periphery of breast carcinomas and peritumor lymphatic vessel density, suggesting that the invasive edge of breast cancers may play a more important role in inducing tumor-associated lymphangiogenesis compared to the rest of the tumors. Recently, others have also demonstrated an association between VEGF-C expression and increased lymphatic vessel density in clinical samples of invasive breast carcinomas. ${ }^{15,30,31}$ In our cohort of invasive breast cancer patients, high VEGF-C expression at the invasive edge, but not the central portions of tumors correlated with poor overall and recurrence-free survival. In recent studies of breast, pancreatic and cervix carcinomas, high VEGF-C expression at the invasive edge of the tumors was also associated with lymphatic invasion and nodal metastases. ${ }^{19,20,29}$

As we have reported previously, ${ }^{16,17}$ we found that the presence of extensive retraction clefts in invasive breast carcinomas strongly correlated with lymphatic tumor spread and poor outcome. We have previously proposed that retraction clefts seen around tumor cell nests and glands in invasive breast carcinomas is likely a morphological reflection of altered tumor-stromal interactions, which may contribute to tumor progression and metastasis observed clinically in these patients. Although currently most investigators consider tumor-associated peritumor lymphatic vessels to be pre-existing vessels, Barsky and Alpaugh ${ }^{32,33}$ have postulated that tumor cell spheroids (tumor cell emboli) may induce the formation of a lymphatic channel around themselves. It is currently accepted that tumorstroma interactions play a significant role in tumor development and progression, and alterations in the stromal microenvironment, such as enhanced vasculature, modified extracellular matrix composition, inflammatory cells and unbalanced protease activity, are essential regulatory factors of tumor growth and invasion. ${ }^{34}$ Data also indicate that tumor cells can regulate the development of a 'tumor stroma' via the aberrant expression of growth factors or induction of growth factor receptors in the stromal component, whereas upon its induction, the tumor stroma will reciprocally influence the tumor cells contributing to the maintenance of a malignant phenotype. $^{34}$

Hartveit $^{35}$ has previously proposed that the breast stroma contains a 'hidden' lymphatic system, formed by attenuated cells that may give origin to stromal channels connected to the lymphatics. It was also suggested that there may be a relationship between these 'pre-lymphatic channels' and the spaces seen in pseudoangiomatous stromal hyperplasia, which, similar to retraction clefts, are apparent on frozen sections and are considered to represent real spaces as opposed to an artifactual phenomenon by several investigators. ${ }^{36,37}$ Damiani et $a .^{36}$ have described malignant neoplasms in the breast that appeared to spread through the spaces of pseudoangiomatous stromal hyperplasia supporting the view that these spaces are real, likely represent the 'pre-lymphatic channels' of Hartveit and suggested that they may represent a previously unrecognized pathway of tumor spread. Given the observed association of the extensive presence of retraction clefts with lymphangiogenesis, as measured by lymphatic vessel density and VEGF-C expression, and lymphatic tumor spread, we propose that retraction clefts are real spaces, likely related to the 'pre-lymphatic channels' of Hartveit, ${ }^{35}$ which may represent an early stage of lymphatic invasion. Growth factors secreted by the tumors cells (especially at the invasive edge of cancers) may stimulate tumor-associated lymphangiogenesis by promoting the endothelialization of these 'prelymphatic channels'. This hypothesis also appears to be supported by the occasional presence of partially formed endothelial lining, as detected by podoplanin immunostaining, in association with retraction clefts surrounding tumor cell nests, suggestive of an early stage of lymphatic invasion (Figure 2). In addition, this hypothesis is further supported by our finding of the apparent similar prognostic significance of extensive retraction clefts and overt lymphatic invasion in breast cancers. Others have also suggested that retraction clefts may in fact be an early stage of lymphatic invasion, where the conversion of mesenchymal cells to endothelial cells has not yet been completed. ${ }^{33}$

We found podoplanin expression in the tumorassociated stroma in 53\% of invasive breast carcinomas in our series, in contrast to lack of expression in the stroma of benign breast tissue. Podoplanin expression has also been observed in fibroblasts during chronic inflammation, ${ }^{38}$ and Kawase et al. ${ }^{39}$ found podoplanin expression within tumor stroma in $30.5 \%$ of invasive adenocarcinomas of the lung, but not in any non-invasive bronchioalveolar carcinomas. In their study, podoplanin expression in tumor stroma significantly correlated with several aggressive tumor features, including larger tumor size, nodal involvement, advanced stage, poor differentiation, vascular and pleural invasion, and predicted shorter survival time. Similarly, we also found that podoplanin expression in tumor stroma of invasive breast carcinomas significantly correlates with aggressive tumor features, such as high histological grade, presence of lymphatic invasion, lack of ER expression and more advanced stage. Interestingly, stromal podoplanin expression also showed a significant correlation with the extent of retraction clefts, high lymphatic vessel density and VEGF-C expression in the tumors.

Fibroblasts recruited into cancer tissue, called cancer-associated fibroblasts, can produce collagens and extracellular matrix proteins in response to several extracellular stimuli and influence cancer cell progression. ${ }^{40}$ Podoplanin expression on cancer 
cells was previously shown to play a role in platelet aggregation and to promote tumor cell adhesion to vascular endothelium, extravasation, metastasis and malignant progression. ${ }^{41,42}$ Our findings suggest that podoplanin may be a biological marker of active fibroblasts and cancer-associated fibroblasts expressing podoplanin may contribute to the progression and aggressive behavior of breast carcinomas.

In summary, our results suggest that the presence of extensive retraction clefts in breast carcinomas highly significantly correlates with lymphangiogenesis (as determined by lymphatic vessel density and VEGF-C expression), lymphatic tumor spread and poor outcome. Further, our results are consistent with the hypothesis that retraction clefts are real spaces, which may represent an early stage of lymphatic invasion and that growth factors secreted by the tumor cells may stimulate tumor associated lymphangiogenesis by promoting the endothelialization of these 'pre-lymphatic channels'.

\section{Acknowledgement}

This study was supported by a DuBose-McLeod Foundation Award and Research Account Funds from the Moffitt Cancer Center (GA). This original research was presented in part at the 97th Annual Meeting of the United States and Canadian Academy of Pathology, Denver, CO, 1-7 March 2008.

\section{Disclosure/conflict of interest}

The authors declare no conflict of interest.

\section{References}

1 Stacker SA, Achen MG, Jussila L, et al. Lymphangiogenesis and cancer metastasis. Nat Rev Cancer 2002;2:573-583.

2 Clarijs R, Ruiter DJ, de Waal RM. Lymphangiogenesis in malignant tumours: Does it occur? J Pathol 2001;193:143-146.

3 Pepper MS. Lymphangiogenesis and tumor metastasis: myth or reality? Clin Cancer Res 2001;7:462-468.

4 Beasley NJ, Prevo R, Banerji S, et al. Intratumor lymphangiogenesis and lymph node metastasis in head and neck cancer. Cancer Res 2002;62:1315-1320.

5 Sipos B, Klapper W, Kruse ML, et al. Expression of lymphangiogenic factors and evidence of intratumor lymphangiogenesis in pancreatic endocrine tumors. Am J Pathol 2004;165:1187-1197.

6 Schoppmann SF, Birner P, Studer P, et al. Lymphatic microvessel density and lymphatic invasion assessed by anti-podoplanin immunostaining in human breast cancer. Anticancer Res 2001;21:2351-2355.

7 Straume O, Jackson DG, Akslen LA. Independent prognostic impact of lymphatic vessel density and presence of low-grade lymphangiogenesis in cutaneous melanoma. Clin Cancer Res 2003;9:250-256.
8 Maula SM, Luukkaa M, Grenman R, et al. Intratumor lymphatics are essential for the metastatic spread and prognosis in squamous cell carcinomas of the head and neck region. Cancer Res 2003;63:1920-1926.

9 Alitalo K, Carmeliet P. Molecular mechanisms of lymphangiogenesis in health and disease. Cancer Cell 2002;1:219-227.

10 Mandriota SJ, Jussila L, Jeltsch M, et al. Vascular endothelial growth factor-C-mediated lymphangiogenesis promotes tumour metastasis. EMBO J 2001;20: 672-682.

11 Skobe M, Hawighorst T, Jackson DG, et al. Induction of tumor lymphangiogenesis by VEGF-C promotes breast cancer metastasis. Nat Med 2001;7:192-198.

12 Karpanen T, Egeblad M, Karkkainen MJ, et al. Vascular endothelial growth factor $\mathrm{C}$ promotes tumor lymphangiogenesis and intralymphatic tumor growth. Cancer Res 2001;61:1786-1790.

13 Mattila MM, Ruohola JK, Karpanen T, et al. VEGF-C induced lymphangiogenesis is associated with lymph node metastasis in orthotopic MCF-7 tumors. Int J Cancer 2002;98:946-951.

14 Nakamura Y, Yasuoka H, Tsujimoto M, et al. Clinicopathological significance of vascular endothelial growth factor-C in breast carcinoma with long-term follow-up. Mod Pathol 2003;16:309-314.

15 Nakamura Y, Yasuoka H, Tsujimoto M, et al. Lymph vessel density correlates with nodal status, VEGF-C expression, and prognosis in breast cancer. Breast Cancer Res Treat 2005;91:125-132.

16 Acs G, Dumoff KL, Solin LJ, et al. Extensive retraction artifact correlates with lymphatic invasion and nodal metastasis and predicts poor outcome in early stage breast carcinoma. Am J Surg Pathol 2007;31:129-140.

17 Acs G, Paragh G, Chuang ST, et al. The presence of micropapillary features and retraction artifact in core needle biopsy material predicts lymph node metastasis in breast carcinoma. Am J Surg Pathol 2009;33:202-210.

18 Pinder SE, Elston CW, Ellis IO. Invasive carcinomausual histologic types. In: Elston CW, Ellis IO (eds). The Breast, 3rd edn. Churchill Livingstone: Edinburgh, 1998,pp 283-337.

19 Gombos Z, Xu X, Chu CS, et al. Peritumor lymphatic vessel density and vascular endothelial growth factor $\mathrm{C}$ expression in early-stage squamous cell carcinoma of the uterine cervix. Clin Cancer Res 2005;11: 8364-8371.

20 Kurahara H, Takao S, Maemura K, et al. Impact of vascular endothelial growth factor-C and -D expression in human pancreatic cancer: its relationship to lymph node metastasis. Clin Cancer Res 2004;10:8413-8420.

21 Weidner N, Semple JP, Welch WR, et al. Tumor angiogenesis and metastasis-correlation in invasive breast carcinoma. N Engl J Med 1991;324:1-8.

22 Nathanson SD, Zarbo RJ, Wachna DL, et al. Microvessels that predict axillary lymph node metastases in patients with breast cancer. Arch Surg 2000;135: 586-593.

23 Williams CS, Leek RD, Robson AM, et al. Absence of lymphangiogenesis and intratumoural lymph vessels in human metastatic breast cancer. J Pathol 2003;200:195-206.

24 Bono P, Wasenius VM, Heikkila P, et al. High LYVE-1positive lymphatic vessel numbers are associated with poor outcome in breast cancer. Clin Cancer Res 2004;10:7144-7149. 
25 Kinoshita J, Kitamura K, Kabashima A, et al. Clinical significance of vascular endothelial growth factor-C (VEGF-C) in breast cancer. Breast Cancer Res Treat 2001;66:159-164.

26 El-Gohary YM, Metwally G, Saad RS, et al. Prognostic significance of intratumor and peritumor lymphatic density and blood vessel density in invasive breast carcinomas. Am J Clin Pathol 2008;129: 578-586.

27 Schoppmann SF, Birner P, Stockl J, et al. Tumorassociated macrophages express lymphatic endothelial growth factors and are related to peritumor lymphangiogenesis. Am J Pathol 2002;161:947-956.

28 Birner P, Schindl M, Obermair A, et al. Lymphatic microvessel density as a novel prognostic factor in early-stage invasive cervical cancer. Int J Cancer 2001;95:29-33.

$29 \mathrm{Gu} \mathrm{Y,} \mathrm{Qi} \mathrm{X,} \mathrm{Guo} \mathrm{S.} \mathrm{Lymphangiogenesis} \mathrm{induced} \mathrm{by}$ VEGF-C and VEGF-D promotes metastasis and a poor outcome in breast carcinoma: a retrospective study of 61 cases. Clin Exp Metast 2008;25:717-725.

30 Choi WW, Lewis MM, Lawson D, et al. Angiogenic and lymphangiogenic microvessel density in breast carcinoma: correlation with clinicopathologic parameters and VEGF-family gene expression. Mod Pathol 2005;18:143-152.

$31 \mathrm{Li}$ YS, Kaneko M, Amatya VJ, et al. Expression of vascular endothelial growth factor-C and its receptor in invasive micropapillary carcinoma of the breast. Pathol Int 2006;56:256-261.

32 Barsky S, Alpaugh M. The molecular basis of inflammatory breast carcinoma [abstract]. Proc Am Assoc Cancer Res 2002;43:368.
33 Barsky SH, Ye Y, Karlin NJ. 'Separation artifact' $v$ lymphatic invasion: Are mimics only mimics? (abstract). Mod Pathol 2006;19 (Suppl. 1):289A.

34 Ingber DE. Cancer as a disease of epithelial-mesenchymal interactions and extracellular matrix regulation. Differentiation 2002;70:547-560.

35 Hartveit E. Attenuated cells in breast stroma: the missing lymphatic system of the breast. Histopathology 1990;16:533-543.

36 Damiani S, Eusebi V, Peterse JL. Malignant neoplasms infiltrating pseudoangiomatous' stromal hyperplasia of the breast: an unrecognized pathway of tumour spread. Histopathology 2002;41:208-215.

37 Vuitch MF, Rosen PP, Erlandson RA. Pseudoangiomatous hyperplasia of mammary stroma. Hum Pathol 1986;17:185-191.

38 Muller AM, Franke FE, Muller KM. D2-40: a reliable marker in the diagnosis of pleural mesothelioma. Pathobiology 2006;73:50-54.

39 Kawase A, Ishii G, Nagai K, et al. Podoplanin expression by cancer associated fibroblasts predicts poor prognosis of lung adenocarcinoma. Int J Cancer 2008;123:1053-1059.

40 Kalluri R, Zeisberg M. Fibroblasts in cancer. Nat Rev Cancer 2006;6:392-401.

41 Wicki A, Lehembre F, Wick N, et al. Tumor invasion in the absence of epithelial-mesenchymal transition: podoplanin-mediated remodeling of the actin cytoskeleton. Cancer Cell 2006;9:261-272.

42 Mahalingam M, Ugen KE, Kao KJ, et al. Functional role of platelets in experimental metastasis studied with cloned murine fibrosarcoma cell variants. Cancer Res 1988;48:1460-1464. 